\title{
COVID-19 and Influenza: Differences, Similarities, and Coinfection
}

\author{
Fariba Shirvani ${ }^{1}$, Delara Babaie (iD) ${ }^{2}$ and Ahmad Moein Karimi ${ }^{1, *}$ \\ ${ }^{1}$ Pediatric Infections Research Center, Research Institute for Children's Health (RICH), Shahid Beheshti University of Medical Sciences, Tehran, Iran \\ ${ }^{2}$ Department of Allergy and Immunology, Mofid Children Hospital, Tehran, Iran \\ "Corresponding author: Pediatric Infections Research Center, Research Institute for Children's Health (RICH), Shahid Beheshti University of Medical Sciences, Tehran, Iran. \\ Email: ahmadmoeinkarimi@gmail.com
}

Received 2021 May 10; Revised 2021 July 24; Accepted 2021 November 07.

\begin{abstract}
Context: COVID-19 and influenza coinfection may increase mortality and morbidity during the COVID-19 pandemic. Recognizing the differences and similarities between COVID-19 and influenza helps us diagnose and treat these 2 diseases. Accordingly, we aimed to compare virologic, clinical, paraclinical, and radiological features and prophylactic and therapeutic management of SARS-CoV-2 and influenza infections. We also provided an algorithmic approach to the diagnosis and treatment of SARS-CoV-2 and influenza coinfection in children.

Evidence Acquisition: Electronic databases, including Cochrane Collaboration, PubMed, Google Scholar, and EMBASE, were searched for the articles published in English language using the following keywords: "influenza virus," "SARS-CoV-2 virus," "COVID19," “comparison," “coinfection,” “management," "treatment," “antiviral therapy," “vaccines," "children,” and "adults." Boolean operations (AND and OR) were used to refine the search. No date limitation was applied.

Results: SARS-CoV-2 and influenza are both RNA viruses with different receptors. The reproductive rate of SARS-CoV-2 is higher than influenza. Patients with SARS-CoV-2 infection, particularly adults, have higher rates of anosmia/ageusia. Organ involvement occurs more frequently in COVID-19 cases, and multisystem inflammatory syndrome in children (MIS-C) occurs especially in children. Disease severity, excessive immune response, and mortality are higher in SARS-CoV-2. Radiological peripheral lesions and ground-glass appearance are characteristic of COVID-19 infection. It is important to rule out influenza and SARS-CoV-2 infection in patients with respiratory problems during the pandemic. Timely prescription of currently available antiviral drugs is essential.

Conclusions: Treatment of patients suspected of having a coinfection is determined by the patient's condition and polymerase chain reaction (PCR) evaluation.
\end{abstract}

Keywords: COVID-19, Influenza virus, Coinfection, Children

\section{Context}

On 31 December 2019, a cluster of pneumonia cases was reported in Wuhan City, China. The disease, now identified as COVID-19 (coronavirus disease 2019), spreads fast and continuously worldwide, with mortality exceeding 4.8 million (1). As the COVID-19 pandemic is evolving continuously, it is important to compare this respiratory virus with other viruses circulating in the community.

Both COVID-19 and influenza are respiratory viruses; however, there are important differences between them in terms of their pathogenesis and clinical course. It has been shown that with the onset of the cold season, there might be a pathogenic interaction between SARS-CoV-2 and influenza viruses, leading to an increase in COVID-19 severity $(2,3)$. Since influenza is a treatable disease, identifying 2 viruses at the same time can play an important role in our treatment plan.

\section{Objectives}

In this review article, we aimed to compare virologic, clinical, paraclinical, and radiological features and prophylactic and therapeutic management of SARS-CoV-2 and influenza infections. We also provided an algorithmic approach to the diagnosis and treatment of SARS-CoV-2 and influenza coinfection in children.

\section{Evidence Acquisition}

\subsection{Search Strategy}

Electronic databases, including Cochrane Collaboration, PubMed, Google Scholar, and EMBASE, were searched for the articles published in English language using the following keywords: "influenza virus," "SARS-CoV-2 virus," “COVID-19," "comparison," "coinfection," "management," "treatment," “antiviral therapy," “vaccines," “children," and 
“adults." Boolean operations (AND and OR) were used to refine the search. No date limitation was applied. Persian databases were searched to retrieve information about influenza and COVID-19 coinfection in Iran.

\subsection{Study Selection}

We included all study types in our search strategy. NO "methodological filter" was applied. Articles were selected for their relevance to the subject (ie, different aspects of influenza and COVID-19 infections). Citations of retrieved articles were checked for additional references to improve our search strategy.

\subsection{Data Extraction}

Multiple observers were used for independent extraction. After data acquisition, 113 articles were selected, 7 articles were duplicated, and 55 were not relevant. We selected 35 original articles, 5 review articles, 1 case series, 4 systematic reviews and meta-analyses, 2 guidelines, 1 correspondence, and 2 articles as an algorithm. Data classification and categorization of findings were performed by a systematic strategy in a meaningful manner. Figure 1 shows the reviewed, eligible, included, and excluded articles.

\section{Results}

\subsection{Virologic Characteristics of Influenza vs SARS-CoV-2}

Both SARS-CoV-2 and influenza viruses are enveloped, single-stranded RNA viruses encapsidated by the nucleoprotein. However, they differ in polarity and segmentation of their genomes. SARS-CoV-2 comprises nonsegmented, positive-sense RNA, while the influenza virus has 8 negative-sense RNA segments. Moreover, SARS-CoV2 has only 1 strain, but influenza has 4 strains (A, B, C, and $D)$ and many subtypes. Using its spike (S) proteins, the SARS-CoV-2 virus binds to angiotensin-converting enzyme 2 (ACE2) expressed on the epithelia of different organs, including the lungs, heart, brain, liver, and intestine. SARSCov-2 RNA is injected into the host cell, and replication of the genome occurs. In the case of the influenza virus, the hemagglutinin (HA) surface protein binds to sialic acid located on host respiratory epithelial cells. HA binds to sialic acid, and the virus enters the host cell. Upon entry, viral RNA is released in host cells, and new virus particles are copied, synthesized, and released (4).

4.2. Transmissibility, Risk Population, Basic Reproductive Rate, and Mortality of Influenza vs SARS-CoV-2

The transmission speed is also a point of difference between the 2 viruses. Compared to COVID-19, influenza has a shorter incubation period ( 2 vs 4 - 12 days). The average basic reproductive number $\left(\mathrm{R}_{0}\right.$; defined as the average number of secondary transmissions from 1 infected person) of SARS-CoV-2 is 2.5 compared to 2 for the 1918 influenza pandemic and 1.7 for the 2009 influenza pandemic (5). It has been shown that newer variants of SARS-CoV-2 (eg, the B.1.1.7 variant in the United Kingdom) are more transmissible than the previous lineage, but no clear changes in disease severity have been reported. Children, the elderly, immunosuppressed patients, and those with chronic medical conditions are the most at risk for severe influenza. Our current knowledge is that underlying conditions and old age increase the risk of severe infection in COVID-19 patients, but children and pregnant women are not at additional risk (6). The crude mortality ratio (the number of death reports divided by reported cases) for COVID-19 is higher than seasonal influenza (2.6\% vs. $0.096 \%)$ (4).

\subsection{Clinical Characteristics of Influenza vs SARS-CoV-2}

Influenza and SARS-CoV-2 share similar clinical features, making it difficult to distinguish between them solely based on symptoms. Fever and cough are the 2 most common symptoms in both seasonal influenza and COVID19 (7). However, there are differences regarding the magnitude and frequency of symptoms between seasonal influenza and COVID-19.

In children, fever, diarrhea or vomiting, headache, body ache or myalgia, and chest pain were significantly more frequent in seasonal influenza, but cough and shortness of breath had a similar frequency (8). A comparative study in France showed that fatigue, faintness, diarrhea, and anosmia/ageusia were significantly more common in adults with COVID-19, but sputum production and nasal congestion were more frequent in those with influenza. Respiratory and other organ involvement are more prominent in COVID-19 cases. Multisystem inflammatory syndrome in children (MIS-C) specifically occurs in children with COVID-19 (9).

\subsection{Co-pathogens in Influenza vs SARS-CoV-2}

Bacterial, viral, and fungal co-pathogens are commonly encountered in viral respiratory tract infections. In severe influenza, bacterial coinfection rate may reach up to $20-30 \%$, being a major cause of morbidity and mortality $(10,11)$. As a result, the American Thoracic Society and Infectious Diseases Society of America have recommended initial antibacterial treatment for adults with communityacquired pneumonia and positive influenza test (12). According to a systematic review and meta-analysis, Streptococcus pneumoniae and Staphylococcus aureus account for most co-pathogens in influenza; however, other coinfecting bacteria are also reported (13). 


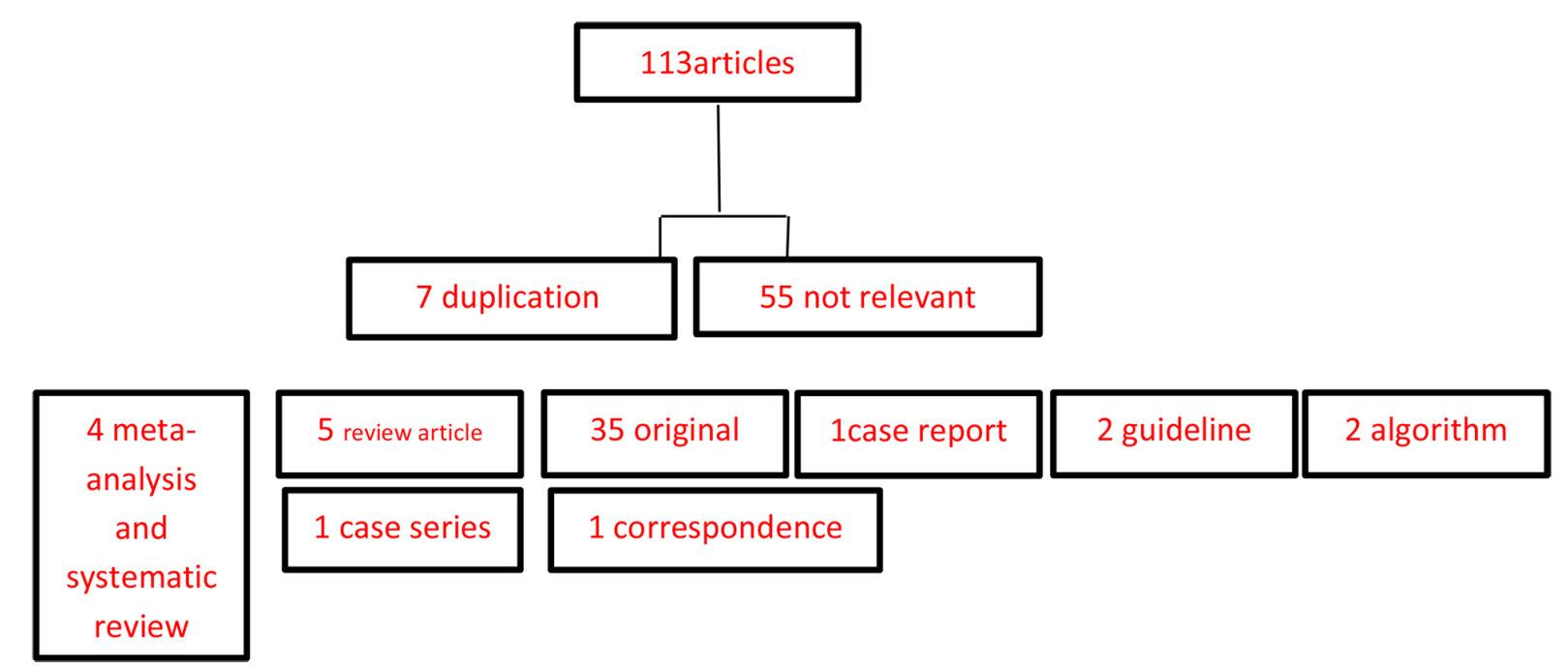

Figure 1. Reviewed, eligible, included, and excluded articles

Studies have reported variable rates of coinfection among COVID-19 patients. A study from Singapore reported no coinfection in COVID-19 patients, while 50\% of COVID-19 non-survivors had coinfections in China (14, 15). The overall bacterial coinfection rate in COVID-19 patients was reported as $7 \%$; the commonest bacteria were Mycoplasma pneumoniae, Pseudomonas aeruginosa, and Haemophilus influenzae. However, Legionella pneumophila, S. aureus, S. pneumoniae, and Chlamydophila pneumoniae are also frequently reported (16). In a study from China, 39 organisms were studied in hospitalized patients with COVID-19, and in $94.2 \%$ of cases, 24 co-pathogens were identified. Streptococcus pneumoniae was the most common, followed by H. influenzae and Klebsiella pneumoniae. The proportion of bacterial, fungal, and viral coinfection was higher in severe cases (17). According to a recent systematic review and meta-analysis, the overall viral coinfection rate in COVID-19 patients was 3\% (18).

In addition to the respiratory syncytial virus and influenza A virus (which are the commonest coinfecting viruses), rhinovirus/enterovirus, adenovirus, parainfluenza, metapneumovirus, and influenza B virus have also been reported in patients with COVID-19 $(8,9)$. Viremia with Epstein-Barr virus (EBV), cytomegalovirus (CMV), and human-herpes virus-6 (HHV-6) were respectively reported in $15 \%, 82 \%$, and $22 \%$ of COVID-19 patients admitted to the intensive care unit (ICU), with EBV viremia associated with longer ICU admission (19). Less commonly, fungal pathogens such as Candida albicans, Aspergillus flavus, A. fumigatus, and C. glabrata were detected in samples taken from COVID-19 patients $(16,18)$.

\subsection{Disease Severity in Influenza vs SARS-CoV-2}

The pathogenesis of influenza A infection occurs in 2 stages. The first stage, which lasts for 1-3 days, is characterized by increased inflammation and determines the peak viral titer. In the second stage, the disease may progress to acute respiratory distress syndrome (ARDS) and even death but at lower rates compared to COVID-19. As a highly pathogenic human coronavirus, SARS-CoV-2 can cause severe lower respiratory tract infection and ARDS; it can also damage other organs, including the gastrointestinal tract, brain, thyroid, skeletal muscle, heart, and lymph nodes, especially in older patients ( $>65$ years old). Moreover, it may cause an excessive immune response named cytokine storm, which in turn may facilitate viral entry into host cells by enhancing the infiltration of non-neutralizing antiviral proteins (20).

\subsection{Imaging in Influenza vs SARS-CoV-2}

Due to its higher resolution and the ability to provide anatomic details, chest computed tomography (CT) is considered a valuable tool in the diagnosis of viral pneumonia, including COVID-19 and influenza. The sensitivity of chest CT in detecting COVID-19 at initial presentation is estimated as 56 - 98\%, higher than reverse transcriptasepolymerase chain reaction (RT-PCR); thus, chest CT can be used in patients who have negative RT-PCR in the initial presentation (21, 22). However, CT features of COVID-19 can also be detected in other viral pneumonia (eg, influenza), making it difficult to distinguish them radiologically (23). Bai et al reported that radiologists could not differentiate 
COVID-19 from other viral pneumonia in about $26 \%$ of cases based on chest CT findings (24).

Although bilateral ground-glass opacity (GGO) and multifocal consolidation are considered the radiologic hallmarks of COVID-19, they can be similarly found in influenza and SARS-CoV-2 $(24,25)$. However, lesions are predominantly peripheral and more located within the lobes in COVID-19, while they are more widespread in influenza. Crazy-paving pattern and well-defined reticular changes were more reported in COVID-19, while pleural effusion, pneumothorax, pneumomediastinum, and centrilobular or "tree-in-bud" nodules are less reported (25).

\subsection{Coinfection of Influenza and SARS-CoV-2}

Cases of influenza and COVID-19 coinfections have been reported in adults from Iran, Spain, China, Germany, Japan, and the USA (26). Although severely ill COVID-19 patients can have concomitant infections with viral and bacterial agents, coinfection with influenza accounts for a higher proportion of deaths (27).

Advanced technology can be used to detect SARS-CoV2 and influenza coinfection. Direct fluorescent antibody (DFA) was used to screen COVID-19 patients for influenza virus but had a lower sensitivity and specificity than RTPCR (26). However, RT-PCR can produce false-negative or false-positive results due to factors such as personnel skills in performing technical procedures and laboratory standards. Multiplex PCR is recommended in high-risk patients with coinfection to decide anti-influenza treatment, but it is not available in low-income countries (28). The reasons for rare dual or multiple concurrent viral infections are recourse competition, use of common receptors, inoculum size differences, and viral interference (29). Currently, there are no reports focusing on the significance of elevation of inflammatory markers in influenza and COVID-19 coinfection. In small series, it was found that there was no direct relationship between coinfection and disease severity, and there might be other reasons affecting the outcome of patients with dual infection $(30,31)$.

\subsection{Antiviral Treatment in Influenza vs SARS-CoV-2}

Antiviral treatment for influenza is recommended in hospitalized children with severe or complicated diseases or those at risk of complications. There are various effective medications used to treat influenza A (pandemic strain $2009 \mathrm{H} 1 \mathrm{N1}$ ) and B, including baloxavir (an inhibitor of mRNA synthesis) and neuraminidase inhibitors (eg, oseltamivir, peramivir, and zanamivir) preventing the virion release from the host cells $(32,33)$. Amantadine (an inhibitor of influenza A M2 ion channels) is no longer recommended for the treatment of influenza due to drug resistance (32).
Different antiviral drugs have been used in COVID-19, including remdesivir, favipiravir, atazanavir, and lopinavirritonavir (34). Despite the lack of data in the treatment of children with COVID-19, remdesivir is preferred in the critical phase of the disease. The triphosphate form of remdesivir (RDV-TP) is used as a substrate in many RNAdependent RNA polymerase complexes and inhibits RNA synthesis in coronavirus. Lopinavir-ritonavir is a protease inhibitor used for the HIV treatment regimen in children but is not recommended for routine use given its unfavorable pharmacodynamics (35). Hydroxychloroquine, a potential antiviral drug, blocks the sialic acid receptors, restricts PH-mediated S protein cleavage at the ACE2 binding site, and prevents cytokine storm. The efficacy of this drug in COVID-19 infection is not clear and has not been licensed for treatment (36).

\subsection{Prophylaxis in Influenza vs SARS-CoV-2}

Chemoprophylaxis significantly diminishes illness among contacts with confirmed influenza cases; however, it is important to consider immunization as a primary way of protection (37). Chemoprophylaxis is recommended in immunocompromised and unimmunized children (those at high risk of complications during the 2 weeks after influenza immunization). In children at high risk of complications, their family members and close contacts should also receive chemoprophylaxis when circulating strains of the influenza virus are not matched by vaccine strains. Post-exposure prophylaxis is indicated in the first 48 hours of exposure with a confirmed or suspected case of influenza 1 day before symptom onset until 24 hours after fever in children at high risk for influenza complications; the most commonly used drugs are oseltamivir and zanamivir (38). Since there is a lack of data on the efficacy of antiviral drugs in the management of COVID-19 in children, no drug is recommended for its prophylaxis. Initiating treatment in the early stages of the disease is the only way to prevent complications in patients with COVID-19 infection (35).

\subsection{Vaccines in Influenza vs SARS-CoV-2}

The influenza vaccine, made and supplied based on the genetic changes of the inactivated, split, and liveattenuated virus, has been administered annually for a long time (32). SARS-CoV-2 vaccines are developed by whole-microbe (live-attenuated, inactivated, and viral vector vaccines), sub-unit, and genetic (DNA and RNA vaccines) approaches (39). New platforms such as DNA and RNA vaccines are employed in a licensed vaccine for the first time. DNA vaccines consist of a target gene and mammalian expression promotors in plasmid DNA. They need 
electroporators as special delivery devices to reach the nucleus, be transcribed into mRNA, and generate protein to stimulate the immune response (40). RNA vaccines, the first vaccines produced for SARS-CoV-2, act by directly translating RNA into the target protein in the cell cytoplasm. They need very low temperatures, which may complicate their storage (41).

To date, $26.3 \%$ of the world population has received at least 1 dose of a COVID-19 vaccine, and 3.66 billion doses have been administered globally, with 29.89 million doses administered each day. Only $1 \%$ of people in low-income countries have received at least 1 dose (42). In Iran, a total of 6.53 million doses have been administered, accounting for $2.6 \%$ of the population (43).

\subsection{Management of Patients with Suspected Influenza and COVID-19 Coinfection}

Specific therapy of COVID-19/influenza coinfection depends on the severity of the disease. Influenza patients at risk of complications should be hospitalized and treated with oseltamivir before the test, regardless of the possibility of coinfection with COVID-19. Treatment of patients with COVID-19 includes isolation, supportive care, oxygen or assisted ventilation, antivirals, antibiotics, immunomodulators, and anticoagulants (44). The severity of the disease is directly related to the severity of the cytokine storm. Intravenous immunoglobulin (IVIG), corticosteroid pulse, hemoperfusion, dexamethasone, and other corticosteroids have been tried to control the disease, reporting contradictory results. However, dexamethasone has been found to reduce mortality in those who need assisted ventilation or supplemental oxygen (45).

Remdesivir was approved for the treatment of COVID19 in hospitalized adult and pediatric patients (more than $40 \mathrm{~kg}$ and $\geq 12$ years). The emergency use authorization allows remdesivir to be used for the treatment of suspected and confirmed COVID-19 in hospitalized patients more than $3.5 \mathrm{~kg}$ (46). Favipiravir has an effective concentration against SARS-CoV-2 infection and is considered an oral formulation for the treatment of mild to moderate COVID-19 in the outpatient setting. This drug reduces the viral load and improves radiological and clinical outcomes (47). Interferons have been used in combination with other drugs or alone in the treatment of COVID-19 (48).

\subsection{Approach to a Child with Symptoms Compatible with Both COVID-19 and Influenza}

Influenza and COVID-19 coinfection is not necessarily associated with an increase in disease severity in mild to moderate cases, but it is important to know that influenza is treatable. The efficacy of influenza vaccines is 40 to $60 \%$; thus, adherence to hygienic protocols, including handwashing, social distancing, and using a mask, are effective preventative measures (49). Figure 2 is an individualized algorithmic approach to the management of children suspected of influenza and COVID-19 coinfection based on clinical manifestation, disease process, response to treatment, and complications.

(1) Suspected cases of COVID-19: Cough, fever, and 3 or more symptoms of fatigue, headache, myalgia, sore throat, nasal running, shortness of breath, anorexia, nausea, vomiting, diarrhea, and loss of consciousness AND residency or work in places with a high risk of transmission during 14 days before the beginning of symptoms, residency or travel to places with community transmission during 14 days before the beginning of symptoms OR severe acute respiratory illness (SARI), including acute respiratory infection needed for the hospital admission. Probable cases of COVID-19: Suspected cases with clinical criteria and history of contact with confirmed or probable cases OR suspected cases with radiological findings compatible with COVID-19 OR patients with a history of anosmia or ageusia (with any cause) OR patients with progressive pneumonia not responding to appropriate treatment OR death without confirmed reasons in a patient with respiratory distress and contact with confirmed or probable cases AND negative or unknown PCR results. Suspected cases of influenza: At least 2 of the following factors: Fever over $38^{\circ} \mathrm{C}$, cough, runny nose, sore throat in the influenza season with or without diarrhea, vomiting, abdominal pain, headache, or irritability with no confirmed reason. Probable cases of influenza: Suspected cases of influenza in the cold season AND a history of close contact with probable/confirmed influenza cases 4 days before the beginning of the illness OR negative or unknown PCR results. Confirmed cases of COVID-19 or influenza: Patients with a positive PCR test for SARS-CoV-2 or influenza (50).

(2) Systolic blood pressure $<2$ SD or $<5$ th percentile for age AND 2 or 3 of the following factors: Tachycardia or bradycardia (heart rate $>160 / \mathrm{min}$ or $<90 / \mathrm{min}$ in infants under 1 year and heart rate $>150$ or $<70$ in older children), capillary refill $>2$ seconds, weak pulse, tachypnea, cold extremities, petechiae or purpura, hyper or hypothermia, oliguria, and raised serum lactate.

(3) The negative pressure isolation room is desired. If not possible, separate beds with a distance of $2 \mathrm{~m}$ and separate equipment (eg, pulse oximeter).

(4) Routine and specific tests to assess organ involvement and MIS-C, imaging, in the case of gastrointestinal symptoms treat dehydration and follow for subsequent symptoms and signs. CT scans can detect SARS-CoV-2 from influenza-but not in all cases. Tests are requested for pa- 


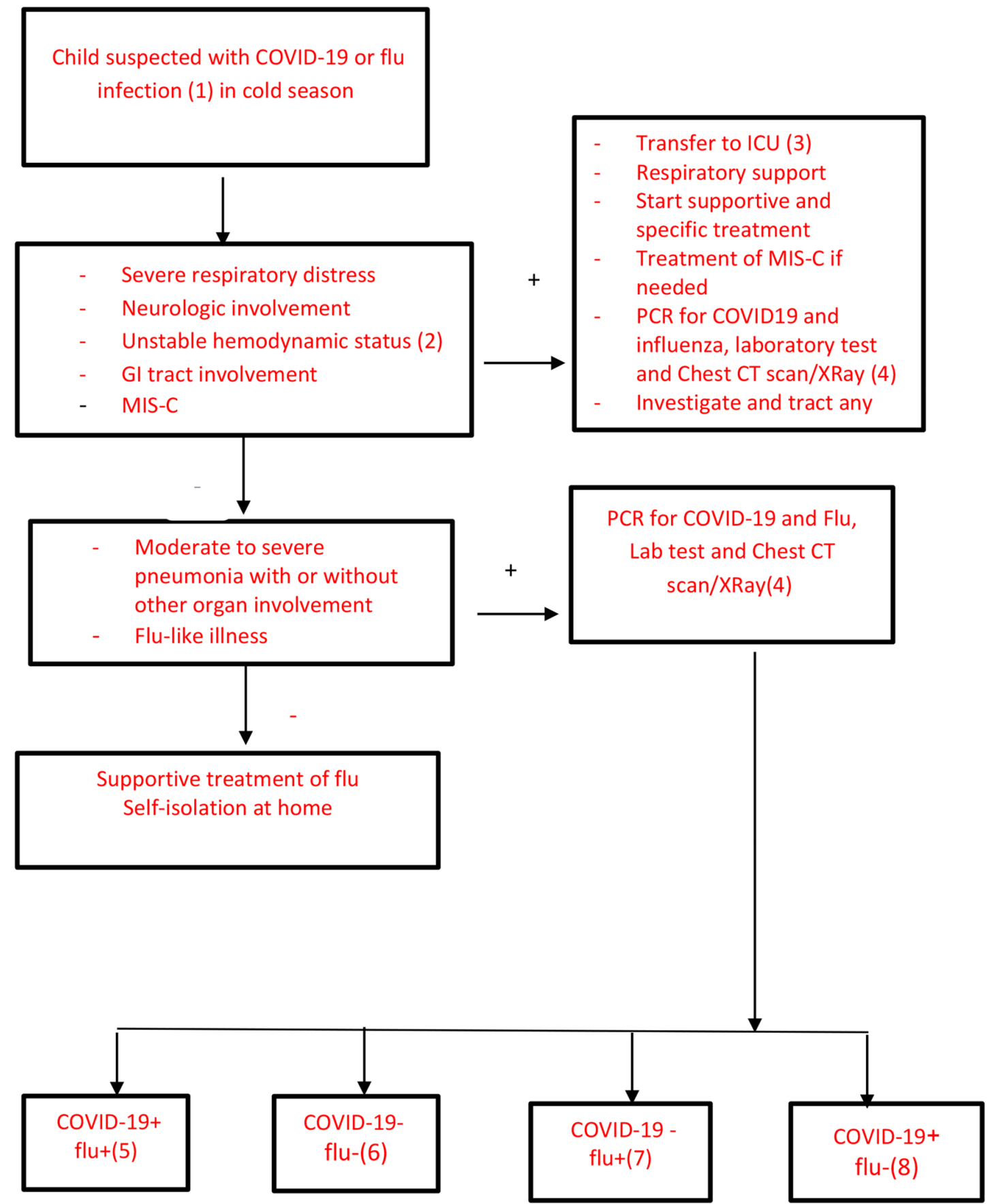

Figure 2. Approach to a child with symptoms compatible with COVID-19 or influenza 
tients according to the organ involvement and the likelihood of MIS-C (51).

(5) Confirmed coinfection: PCR tests for influenza and SARS-CoV-2 are positive. Interferon $\beta$ 1a/interferon $\beta$ 1b, 5 to 7 doses every other day plus oseltamivir for 5 days or favipiravir for 7 - 10 days. If separation is possible, patients should be admitted to COVID-19 and influenza rooms. Postexposure prophylaxis is necessary according to the Iranian influenza algorithm for certain groups : (1) cases at high risk of influenza complications after 2 weeks of vaccination, (2) cases who cannot receive influenza vaccine because of complications, (3) cases with severe immune deficiencies that may not respond to influenza vaccine, and (4) family members/close contacts, health-care professionals, and children who are at high risk for complications when circulating influenza viruses are not matched with seasonal strains of influenza vaccine $(50,51)$.

(6) If PCR tests for influenza and SARS-CoV-2 are both negative, a definite diagnosis cannot be made based on clinical and paraclinical findings. It is recommended to treat the patient as COVID-19. PCR for influenza and COVID19 should be repeated.

(7) If the PCR test is positive for influenza and negative for COVID-19, retesting for COVID-19 is done, and the patient is treated as an influenza case. In patients with possible influenza and/or SARS-Cov 2 infection, treatment can be started before the test results are ready.

(8) If the PCR test is positive for COVID-19 and negative for influenza, the patient should be treated as a COVID-19 case (51).

\section{Conclusions}

Based on what has been reported, we concluded that due to the importance of influenza, it is very important to identify it in COVID-19 patients. Influenza has its own treatment and prophylaxis plan, and if these 2 infections coincide, the treatment plan will change. The severity of coinfection depends on various factors, some of which are related to the characteristics of the host and the characteristics and behaviors of the virus. Guidelines for simultaneous treatment of these 2 diseases can help us to better control, especially in the cold season when this coinfection is more likely to occur.

\section{Footnotes}

Authors' Contribution: F. SH. drafted the manuscript and D. B. and A. M. K. edited it.

Conflict of Interests: There is no conflict of interest.
Funding/Support: This study received no financial support.

\section{References}

1. Chaplin S. COVID-19: a brief history and treatments in development. Prescriber. 2020;31(5). doi: 10.1002/psb.1843.

2. Stowe J, Tessier E, Zhao H, Guy R, Muller-Pebody B, Zambon M, et al. Interactions between SARS-CoV-2 and influenza, and the impact of coinfection on disease severity: a test-negative design. Int J Epidemiol. 2021;50(4). doi: 10.1093/ije/dyab081. [PubMed: 33942104]. [PubMed Central: PMC8135706].

3. Khodamoradi Z, Moghadami M, Lotfi M. Co-infection of Coronavirus Disease 2019 and Influenza A: A Report from Iran. Arch Iran Med. 2020;23(4):239-43. doi:10.34172/aim.2020.04. [PubMed: 32271596].

4. Hagen A. COVID-19 and the Flu. American Society for Microbiology; 2021. Available from: https://asm.org/Articles/2020/July/COVID19-and-the-Flu.

5. Petersen E, Koopmans M, Go U, Hamer DH, Petrosillo N, Castelli F, et al. Comparing SARS-CoV-2 with SARS-CoV and influenza pandemics. Lancet Infect Dis. 2020;20(9):e238-44. doi: 10.1016/s14733099(20)30484-9.

6. Davies NG, Abbott S, Barnard RC, Jarvis CI, Kucharski AJ, Munday JD, et al. Estimated transmissibility and impact of SARS-CoV2 lineage B.1.1.7 in England. Science. 2021;372(6538). doi: 10.1126/science.abg3055. [PubMed: 33658326]. [PubMed Central: PMC8128288].

7. Pormohammad A, Ghorbani S, Khatami A, Razizadeh MH, Alborzi E, Turner RJ, et al. Global Comparison of Influenza Type A and B with COVID-19: A Systematic Review and Meta-Analysis on Clinical, Laboratory, and Radiographic Findings. SSRN Electron J. 2020. doi: 10.2139/ssrn.3605264.

8. Song X, Delaney M, Shah RK, Campos JM, Wessel DL, DeBiasi RL. Comparison of Clinical Features of COVID-19 vs Seasonal Influenza A and B in US Children. JAMA Netw Open. 2020;3(9). e2020495. doi: 10.1001/jamanetworkopen.2020.20495. [PubMed: 32897374]. [PubMed Central: PMC7489826].

9. Faury H, Courboulès C, Payen M, Jary A, Hausfater P, Luyt C, et al. Medical features of COVID-19 and influenza infection: A comparative study in Paris, France. Infection. 2021;82(2):e36-9.

10. Rice TW, Rubinson L, Uyeki TM, Vaughn FL, John BB, Miller R3, et al. Critical illness from 2009 pandemic influenza A virus and bacterial coinfection in the United States. Crit Care Med.2012;40(5):1487-98. doi: 10.1097/CCM.ob013e3182416f23. [PubMed: 22511131]. [PubMed Central: PMC3653183].

11. Shah NS, Greenberg JA, McNulty MC, Gregg KS, Riddell J, Mangino $\mathrm{JE}$, et al. Bacterial and viral co-infections complicating severe influenza: Incidence and impact among 507 U.S. patients, 2013-14. J Clin Virol. 2016;80:12-9. doi: 10.1016/j.jcv.2016.04.008. [PubMed: 27130980]. [PubMed Central: PMC7185824].

12. Metlay JP, Waterer GW, Long AC, Anzueto A, Brozek J, Crothers K, et al. Diagnosis and Treatment of Adults with Community-acquired Pneumonia. An Official Clinical Practice Guideline of the American Thoracic Society and Infectious Diseases Society of America. Am J Respir Crit Care Med. 2019;200(7):e45-67. doi: 10.1164/rccm.201908-1581ST. [PubMed: 31573350]. [PubMed Central: PMC6812437].

13. Klein EY, Monteforte B, Gupta A, Jiang W, May L, Hsieh YH, et al. The frequency of influenza and bacterial coinfection: a systematic review and meta-analysis. Influenza Other Respir Viruses. 2016;10(5):394403. doi: 10.1111/irv.12398. [PubMed: 27232677]. [PubMed Central: PMC4947938].

14. Young BE, Ong SWX, Kalimuddin S, Low JG, Tan SY, Loh J, et al. Epidemiologic Features and Clinical Course of Patients Infected With SARSCoV-2 in Singapore. JAMA. 2020;323(15). doi: 10.1001/jama.2020.3204. [PubMed: 32125362]. [PubMed Central: PMC7054855]. 
15. Zhou F, Yu T, Du R, Fan G, Liu Y, Liu Z, et al. Clinical course and risk factors for mortality of adult inpatients with COVID-19 in Wuhan, China: a retrospective cohort study. Lancet. 2020;395(10229):1054-62. doi: 10.1016/s0140-6736(20)30566-3.

16. Lai CC, Wang CY, Hsueh PR. Co-infections among patients with COVID-19: The need for combination therapy with non-anti-SARSCoV-2 agents? J Microbiol Immunol Infect. 2020;53(4):505-12. doi: 10.1016/j.jmii.2020.05.013. [PubMed: 32482366]. [PubMed Central: PMC7245213].

17. Zhu X, Ge Y, Wu T, Zhao K, Chen Y, Wu B, et al. Co-infection with respiratory pathogens among COVID-2019 cases. Virus Res. 2020;285:198005. doi: 10.1016/j.virusres.2020.198005. [PubMed: 32408156]. [PubMed Central: PMC7213959].

18. Lansbury L, Lim B, Baskaran V, Lim WS. Co-infections in people with COVID-19: a systematic review and meta-analysis. J Infect. 2020;81(2):266-75. doi: 10.1016/j.jinf.2020.05.046. [PubMed: 32473235]. [PubMed Central: PMC7255350].

19. Simonnet A, Engelmann I, Moreau AS, Garcia B, Six S, El Kalioubie A, et al. High incidence of Epstein-Barr virus, cytomegalovirus, and human-herpes virus-6 reactivations in critically ill patients with COVID-19. Infect Dis Now. 2021;51(3):296-9. doi: 10.1016/j.idnow.2021.01.005. [PubMed: 33495765]. [PubMed Central: PMC7816954].

20. Abdelrahman Z, Li M, Wang X. Comparative Review of SARS-CoV-2, SARS-CoV, MERS-CoV, and Influenza A Respiratory Viruses. Front Immunol. 2020;11:552909. doi: 10.3389/fimmu.2020.552909. [PubMed: 33013925]. [PubMed Central: PMC7516028].

21. Fang Y, Zhang H, Xie J, Lin M, Ying L, Pang P, et al. Sensitivity of Chest CT for COVID-19: Comparison to RT-PCR. Radiology. 2020;296(2):E1157. doi: 10.1148/radiol.2020200432. [PubMed: 32073353]. [PubMed Central: PMC7233365].

22. Kanne JP, Little BP, Chung JH, Elicker BM, Ketai LH. Essentials for Radiologists on COVID-19: An Update-Radiology Scientific Expert Panel. Radiology. 2020;296(2):E113-4. doi: 10.1148/radiol.2020200527. [PubMed: 32105562]. [PubMed Central: PMC7233379].

23. Altmayer S, Zanon M, Pacini GS, Watte G, Barros MC, Mohammed T, et al. Comparison of the Computed Tomography Findings in COVID19 and Other Viral Pneumonia in Immunocompetent Adults: A Systematic Review and Meta-Analysis. SSRN Electron J. 2020:1-12. doi: 10.2139/ssrn.3572873.

24. Bai HX, Hsieh B, Xiong Z, Halsey K, Choi JW, Tran TML, et al. Performance of Radiologists in Differentiating COVID-19 from NonCOVID-19 Viral Pneumonia at Chest CT. Radiology. 2020;296(2):E4654. doi: 10.1148/radiol.2020200823. [PubMed: 32155105]. [PubMed Central: PMC7233414].

25. Onigbinde SO, Ojo AS, Fleary L, Hage R. Chest Computed Tomography Findings in COVID-19 and Influenza: A Narrative Review. Biomed Res Int. 2020;2020:6928368. doi: 10.1155/2020/6928368. [PubMed: 32596354]. [PubMed Central: PMC7275219].

26. Ozaras R, Cirpin R, Duran A, Duman H, Arslan O, Bakcan Y, et al. Influenza and COVID-19 coinfection: Report of six cases and review of the literature. J Med Virol. 2020;92(11). doi: 10.1002/jmv.26125. [PubMed: 32497283].

27. Cuadrado-Payán E, Montagud-Marrahi E, Torres-Elorza M, Bodro M, Blasco M, Poch E, et al. SARS-CoV-2 and influenza virus co-infection. Lancet. 2020;395(10236). doi:10.1016/s0140-6736(20)31052-7.

28. Tahamtan A, Ardebili A. Real-time RT-PCR in COVID-19 detection: issues affecting the results. Expert Rev Mol Diagn. 2020;20(5):453-4. doi: 10.1080/14737159.2020.1757437. [PubMed: 32297805]. [PubMed Central: PMC7189409].

29. Calcagno A, Ghisetti V, Burdino E, Trunfio M, Allice T, Boglione L, et al. Co-infection with other respiratory pathogens in COVID-19 patients. Clin Microbiol Infect. 2021;27(2). doi: 10.1016/j.cmi.2020.08.012. [PubMed: 32822882]. [PubMed Central: PMC7434691].

30. Miatech JL, Tarte NN, Katragadda S, Polman J, Robichaux SB. A case series of coinfection with SARS-CoV-2 and influenza virus in Louisiana.
Respir Med Case Rep. 2020;31:101214. doi: 10.1016/j.rmcr.2020.101214. [PubMed: 32923361]. [PubMed Central: PMC7480397].

31. Ding Q, Lu P, Fan Y, Xia Y, Liu M. The clinical characteristics of pneumonia patients coinfected with 2019 novel coronavirus and influenza virus in Wuhan, China. J Med Virol. 2020;92(9):17. doi: 10.1002/jmv.25781. [PubMed: 32196707]. [PubMed Central: PMC7228290].

32. Uyeki TM, Bernstein HH, Bradley JS, Englund JA, File TM, Fry AM, et al. Clinical Practice Guidelines by the Infectious Diseases Society of America: 2018 Update on Diagnosis, Treatment, Chemoprophylaxis, and Institutional Outbreak Management of Seasonal Influenzaa. Clin Infect Dis. 2019;68(6):895-902. doi: 10.1093/cid/ciy874. [PubMed: 30834445]. [PubMed Central: PMC6769232].

33. Heo YA. Baloxavir: First Global Approval. Drugs. 2018;78(6):693-7. doi: 10.1007/s40265-018-0899-1. [PubMed: 29623652].

34. Frediansyah A, Tiwari R, Sharun K, Dhama K, Harapan H. Antivirals for COVID-19: A critical review. Clin Epidemiol Glob Health. 2021;9:908. doi: 10.1016/j.cegh.2020.07.006. [PubMed: 33521390]. [PubMed Central: PMC7831805].

35. Chiotos K, Hayes M, Kimberlin DW, Jones SB, James SH, Pinninti SG, et al. Multicenter Interim Guidance on Use of Antivirals for Children With Coronavirus Disease 2019/Severe Acute Respiratory Syndrome Coronavirus 2. J Pediatric Infect Dis Soc. 2020;10(1). doi: 10.1093/jpids/piaa115. [PubMed: 32918548]. [PubMed Central: PMC7543452].

36. Hernandez AV, Roman YM, Pasupuleti V, Barboza JJ, White CM. Hydroxychloroquine or Chloroquine for Treatment or Prophylaxis of COVID-19: A Living Systematic Review. Ann Intern Med.2020;173(4):28796. doi: 10.7326/M20-2496. [PubMed: 32459529].

37. Uyeki TM, Bernstein HH, Bradley JS, Englund JA, File TM, Fry AM et al. Clinical Practice Guidelines by the Infectious Diseases Society of America: 2018 Update on Diagnosis, Treatment, Chemoprophylaxis, and Institutional Outbreak Management of Seasonal Influenzaa. Clin Infect Dis. 2019;68(6):e1-e47. doi:10.1093/cid/ciy866. [PubMed: 30566567]. [PubMed Central: PMC6653685].

38. Committee on Infectious Diseases. Recommendations for Prevention and Control of Influenza in Children, 2020-2021. Pediatrics. 2020;146(4). doi: 10.1542/peds.2020-024588. [PubMed: 32900875].

39. Krammer F. SARS-CoV-2 vaccines in development. Nature 2020;586(7830):516-27. doi: 10.1038/s41586-020-2798-3. [PubMed: 32967006].

40. Yu J, Tostanoski LH, Peter L, Mercado NB, McMahan K, Mahrokhian SH, et al. DNA vaccine protection against SARS-CoV-2 in rhesus macaques. Science. 2020;369(6505):806. doi: 10.1126/science.abc6284. [PubMed: 32434945]. [PubMed Central: PMC7243363].

41. Edwards KM, Orenstein WA, Hirsch MS, Bloom A. Coronavirus disease 2019 (COVID-19): Vaccines to prevent SARS-CoV-2 infection. UpToDate; 2020. Available from: https://www.uptodate.com/contents/ coronavirus-disease-2019-covid-19-vaccines-to-prevent-sarscov-2-infection.

42. Our World in Data. Coronavirus (COVID-19) Vaccinations. Our World in Data; 2020. Available from: https://ourworldindata.org/covidvaccinations.

43. World Health Organization. Verified information- COVID-19 Vaccine Updates. World Health Organization; 2020. Available from: https://www.who.int/emergencies/diseases/novel-coronavirus2019/covid-19-vaccines.

44. World Health Organization. COVID-19 clinical management: Living guidance. Geneva, Switzerland: World Health Organization; 2020.

45. Horby P, Lim WS, Emberson J, Mafham M, Bell J, Linsell L, et al. Dexamethasone in hospitalized patients with COVID-19-preliminary report. N Engl J Med. 2020;384(8):693-704. doi:10.1101/2020.06.22.20137273.

46. UpToDate. Remdesivir: Pediatric Drug Information. UpToDate; 2022. Available from: https://www.uptodate.com/contents/remdesivirpediatric-drug-information. 
47. Joshi S, Parkar J, Ansari A, Vora A, Talwar D, Tiwaskar M, et al. Role of favipiravir in the treatment of COVID-19. Int J Infect Dis. 2021;102:501-8. doi: 10.1016/j.ijid.2020.10.069. [PubMed: 33130203]. [PubMed Central: PMC7831863].

48. Haji Abdolvahab M, Moradi-Kalbolandi S, Zarei M, Bose D, Majidzadeh AK, Farahmand L. Potential role of interferons in treating COVID-19 patients. Int Immunopharmacol. 2021;90:107171. doi: 10.1016/j.intimp.2020.107171. [PubMed: 33221168]. [PubMed Central: PMC7608019].
49. Karimi A, Rafiei Tabatabaei S, Shiva F, Hoseinialfatemi SM. COVID-19 or Influenza, or Both? AComparison and Algorithmic Approach to Management. Jundishapur J Microbiol. 2020;13(12). doi: 10.5812/jjm.112121.

50. Karimi A, Rafei Tabatabai S, Pourmogadas Z, Rahimi H, Sedigi I, Armin S, et al. Guide to the diagnosis and treatment of COVID 19 in children. 5. 6 th ed. Ministry of Health and Medical Education; 2020.

51. Karimi A, Rafiei Tabatabaei S, Pourmoghaddas Z. Applied clinical algorithm of influenza: Management of influenza in young people.J Iran Med Counc. 2018;1(1):2-6. 